\title{
A New Filter Nonmonotone Adaptive Trust Region Method for Unconstrained Optimization
}

\author{
Xinyi Wang ${ }^{1}$, Xianfeng Ding ${ }^{1,2, * \mathbb{D}}$ and Quan $Q u^{1}$ \\ 1 School of Science, Southwest Petroleum University, Chengdu 610500, China; \\ 201821000481@stu.swpu.edu.cn (X.W.); 201921000533@stu.swpu.edu.cn (Q.Q.) \\ 2 School of Artificial Intelligence, Southwest Petroleum University, Chengdu 610500, China \\ * Correspondence: dingxianfeng@swpu.edu.cn; Tel.: +86-133-0823-8057
}

Received: 17 December 2019; Accepted: 21 January 2020; Published: 2 February 2020

check for updates

\begin{abstract}
In this paper, a new filter nonmonotone adaptive trust region with fixed step length for unconstrained optimization is proposed. The trust region radius adopts a new adaptive strategy to overcome additional computational costs at each iteration. A new nonmonotone trust region ratio is introduced. When a trial step is not successful, a multidimensional filter is employed to increase the possibility of the trial step being accepted. If the trial step is still not accepted by the filter set, it is possible to find a new iteration point along the trial step and the step length is computed by a fixed formula. The positive definite symmetric matrix of the approximate Hessian matrix is updated using the MBFGS method. The global convergence and superlinear convergence of the proposed algorithm is proven by some classical assumptions. The efficiency of the algorithm is tested by numerical results.
\end{abstract}

Keywords: unconstrained optimization; adaptive trust region; nonmonotone; filter; convergence

\section{Introduction}

Consider the following unconstrained optimization problem:

$$
\min _{x \in R^{n}} f(x),
$$

where $f: R^{n} \rightarrow R$ is a twice continuously differentiable function. The trust region method is one of the prominent classes of iterative methods. At the iteration point $x_{k}$, the trial step $d_{k}$ is obtained by the following quadratic subproblem:

$$
\begin{gathered}
\min _{d \in R^{n}} m_{k}(d)=g_{k}^{T} d+\frac{1}{2} d^{T} B_{k} d, \\
\|d\| \leq \Delta_{k}
\end{gathered}
$$

where $\|$.$\| is the Euclidean norm, f_{k}=f\left(x_{k}\right), g_{k}=\nabla f\left(x_{k}\right), G_{k}=\nabla^{2} f\left(x_{k}\right), B_{k}$ is a symmetric approximation of $G_{k}$, and $\Delta_{k}$ is a trust region radius. The most ordinary ratio is defined as follows:

$$
\rho_{k}=\frac{f_{k}-f\left(x_{k}+d\right)}{m_{k}(0)-m_{k}\left(d_{k}\right)}
$$

Generally, the numerator is referred to as the actual reduction and the denominator is known as the predicted reduction.

The disadvantage of the traditional trust region method is that the subproblem needs to be solved many times to achieve an acceptable trial step in one iteration. To overcome this drawback, Mo et al. [1] first proposed a nonmonotone trust region algorithm with a fixed step length. When the trial 
step is not acceptable, we use a fixed step length to find a new iteration point instead of resolving the subproblem. Based on this advantage, Ou, Hang, and Wang have proposed a trust region algorithm with fixed step length in [2-4], respectively. The fixed step length is computed by

$$
\alpha_{k}=-\frac{\delta g_{k}^{T} d_{k}}{d_{k}^{T} B_{k} d_{k}},
$$

It is well known that the strategy of selecting a trust region radius has a significant impact on the performance of the trust region methods. In 1997, Sartenaer [5] presented a strategy which can automatically determine an initial trust region radius. This fact leads to an increase in the number of subproblems to be solved in some problems, thereby reducing the efficiency of these methods. In 2002, Zhang et al. [6] provided another scheme to reduce the number of subproblems that need to be solved, where the trust region radius uses: $\Delta_{k}=c^{p}\left\|g_{k}\right\|\left\|\hat{B}_{k}^{-1}\right\|, \hat{B}_{k}=B_{k}+i I$, where $i \in N$. Zhang's strategy requires an estimation of the inverse of the matrixes $B_{k}$ and $\hat{B}_{k}^{-1}$ in each iteration; however, Li [4] has suggested another practically efficient adaptive trust region radius that uses $\Delta_{k}=\frac{\left\|d_{k-1}\right\|}{\left\|y_{k-1}\right\|}\left\|g_{k}\right\|$. The strategy requires not only the gradient value but also the function value. Inspired by these facts, some modified versions of adaptive trust region methods have been proposed in [7-10].

As we all know, monotone techniques may slow down the rate of convergence, especially in the presence of the narrow curved valley. The monotone techniques require the value of the function to be decreased at each iteration. In order to overcome these disadvantages, Deng et al. [11] proposed a nonmonotone trust region algorithm in 1993 . The general nonmonotone term $f_{l(k)}$ is defined by

$$
f_{l(k)}=f\left(x_{l(k)}\right)=\max _{0 \leq j \leq m(k)}\left\{f_{k-j}\right\} k=0,1,2, \ldots,
$$

where $m(0)=0,0 \leq m(k) \leq \min \{N, m(k-1)+1\}$, and $N \geq 0$ is an integer. Deng et al. [11] modified the ratio (3) which evaluates the consistency between the quadratic model and the objective function in trust region methods. The most common nonmonotone ratio is defined as follows:

$$
\widetilde{\rho}_{k}=\frac{f_{l(k)}-f\left(x_{k}+d_{k}\right)}{m_{k}(0)-m_{k}\left(d_{k}\right)}
$$

The general nonmonotone term $f_{l(k)}$ suffers from various drawbacks, such as the fact that numerical performance is highly dependent on the choice of $N$. In order to introduce a more suitable nonmonotone strategy, Ahookhosh et al. [12] proposed a new nonmonotone ratio as follows.

$$
\hat{\rho}_{k}=\frac{R_{k}-f\left(x_{k}+d_{k}\right)}{m_{k}(0)-m_{k}\left(d_{k}\right)}
$$

where

$$
R_{k}=\eta_{k} f_{l(k)}+\left(1-\eta_{k}\right) f_{k}
$$

in which $\eta_{k} \in\left[\eta_{\min }, \eta_{\max }\right]$, with $\eta_{\min } \in[0,1)$, and $\eta_{\max } \in\left[\eta_{\min }, 1\right]$. We recommend that interested readers refer to $[13,14]$ for more details and progress on the nonmonotone trust region algorithm.

In order to overcome the difficulties associated with using the penalty function, especially the adjustment of the penalty parameter. The filter methods were recently presented by Fletcher and Leyffer [15] for constrained nonlinear optimization. More recently, Gould et al. [16] explored a new nonmonotone trust region algorithm for the unconstrained optimization problems with the multidimensional filter technique in [17]. Compared with the standard nonmonotone algorithm, the new algorithm dynamically determines iterations based on filter elements and increases the possibility of the trial step being accepted. Therefore, this topic has received great attention in recent years (see [18-21]). 
The remainder of this paper is organized as follows. In Section 2, we describe a new trust region method. The global convergence is investigated in Section 3. In Section 4, we prove the superlinear convergence of the algorithm. Numerical results are shown in Section 5. Finally, the paper ends with some conclusions in Section 6.

\section{The New Algorithm}

In this section, we propose a trust region method by combining a new trust region radius and the modified trust region ratio to effectively solve unconstrained optimization problems. In each iteration, a trial step $d_{k}$ is generated by solving an adaptive trust region subproblem,

$$
\begin{gathered}
\min _{d \in \mathbb{R}^{n}} m_{k}(d)=g_{k}^{T} d+\frac{1}{2} d^{T} B_{k} d, \\
\|d\| \leq \Delta_{k}:=c_{k}\left\|g_{k}\right\|^{\gamma},
\end{gathered}
$$

where $0<\gamma<1$ and $c_{k}$ is an adjustment parameter. Prompted by the adaptive technique, the proposed method has the following effective properties: it is not necessary to calculate the matrix of the inverse and the value of each iteration point, and the algorithm also reduces the related workload and calculation time.

In fact, the matrix $B_{k}$ is usually obtained by approximation, and the subproblems are only solved roughly. In this case, it may be more reasonable to adjust the next trust radius $\Delta_{k+1}$, not only according to $\hat{\rho}_{k}$, but also by considering the use of $\left\{\hat{\rho}_{k-m}, \ldots, \hat{\rho}_{k}\right\}$. To improve the efficiency of the nonmonotone trust region methods, we can define the modified ratio formula based on (7) as follows:

$$
\hat{\rho}_{k}^{\prime}=\sum_{i=1}^{\min \{k, m\}} w_{k i} \hat{\rho}_{k-i+1}
$$

where $m$ is a positive integer and $w_{k i}$ is the weight of $\hat{\rho}_{k-i+1}$, such that $\sum_{i=1}^{\min \{k, m\}} w_{k i}=1$.

More exactly, $\hat{\rho}_{k}$ can be used to determine whether the trial step is acceptable. Adjusting the next radius $\Delta_{k+1}$ depends on (11), thus $c_{k}$ is updated by

$$
c_{k+1}=\left\{\begin{array}{ll}
\min \left\{\beta_{2} c_{k}, c_{\max }\right\} & \text { if } \hat{\rho}_{k}{ }^{\prime} \geq \mu_{2} \\
c_{k} & \text { if } \mu_{1} \leq \hat{\rho}_{k}{ }^{\prime}<\mu_{2} \\
\beta_{1} c_{k} & \text { if } \hat{\rho}_{k}{ }^{\prime}<\mu_{1}
\end{array},\right.
$$

In what follows, we refer to $\nabla f\left(x_{k}\right)$ by $g_{k}=g\left(x_{k}\right)$. When an $i-$ th component of $g_{k}$ is needed, we denote it using $g_{i}\left(x_{k}\right)$. Based on this filter, we say that an iterate point $x_{1}$ dominates $x_{2}$ if, and only if

$$
\left|g_{i}\left(x_{1}\right)\right| \leq\left|g_{i}\left(x_{2}\right)\right| \forall i=1,2, \ldots, n .
$$

A multidimensional filter $F$ is a list of $n$-tuples of the form $\left(g_{1}\left(x_{k}\right), g_{2}\left(x_{k}\right), \ldots, g_{n}\left(x_{k}\right)\right)$, such that

$$
\left|g_{j}\left(x_{k}\right)\right| \leq\left|g_{j}\left(x_{l}\right)\right| j \in\{1,2,3, \ldots, n\},
$$

where $g_{k}, g_{l}$ belong to $F$.

For all $g\left(x_{l}\right) \in F$, a new trial point is acceptable if there exists $j \in\{1,2,3, \ldots, n\}$, such that

$$
\left|g_{j}\left(x_{k}\right)\right| \leq\left|g_{j}\left(x_{l}\right)\right|-\gamma_{g}\left\|g\left(x_{l}\right)\right\| \gamma_{g} \in\left(0, \frac{1}{\sqrt{n}}\right)
$$

If the iterate point $x_{k}^{+}$is acceptable, we add $g\left(x_{k}^{+}\right)$to the filter. Meanwhile, we remove all the points which are dominated by $x_{k}^{+}$from the filter. In the general filter trust region algorithm, for the 
trial point $x_{k}^{+}$satisfying $\hat{\rho}_{k}<\mu_{1}$, we verify whether it is accepted by filter $F$. In our algorithm, it is the trial point $x_{k}^{+}$that satisfies $0<\hat{\rho}_{k}<\mu_{1}$, and verifies whether or not it is accepted by the filter $F$.

Our discussion can be summarized as the following Algorithm 1.

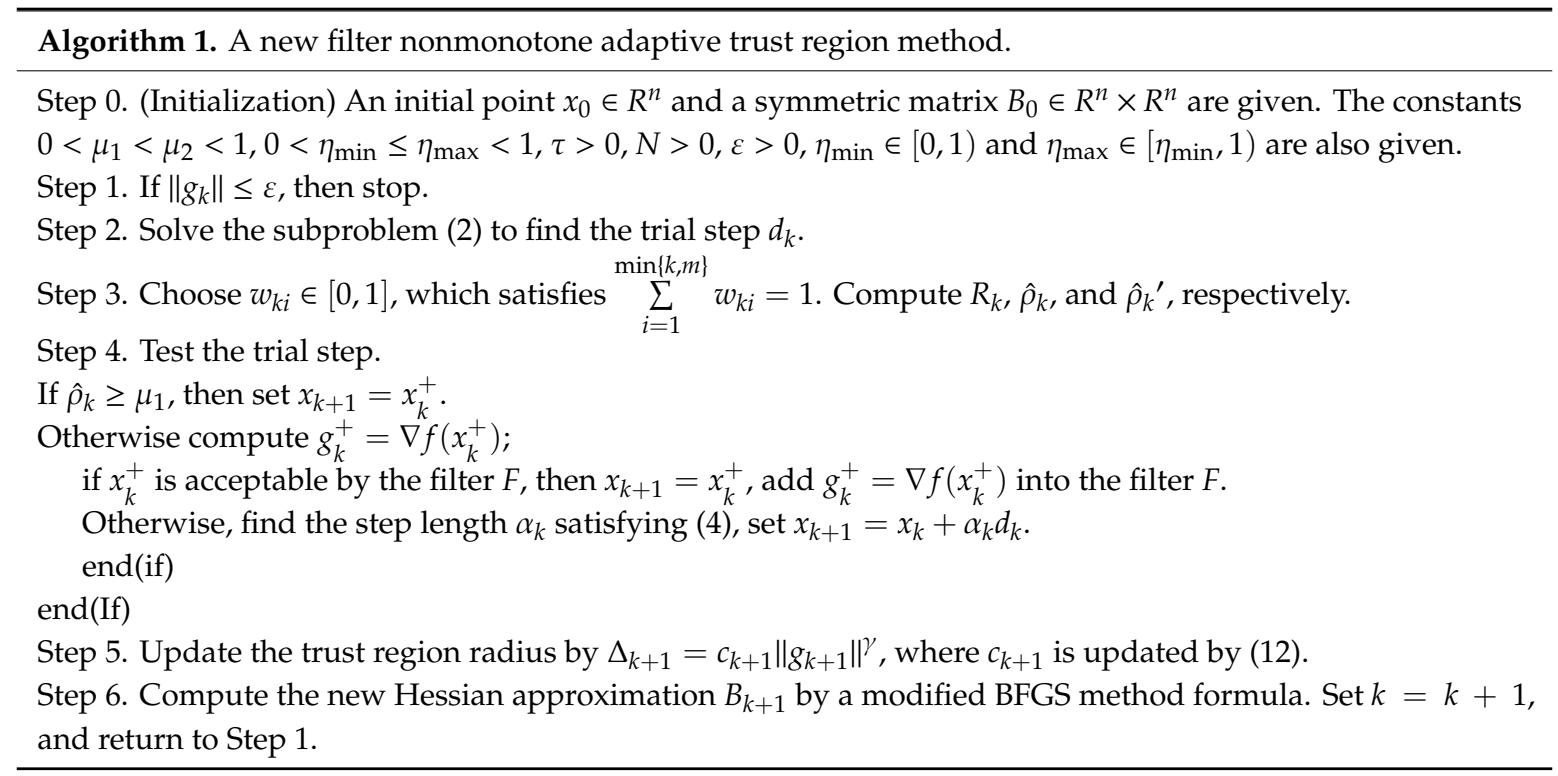

In order to obtain convergence results, we use the following notation:

$D=\left\{k \mid \hat{\rho}_{k} \geq \mu_{1}\right\}, A=\left\{k \mid 0<\hat{\rho}_{k}<\mu_{1}\right.$ and $x_{k}^{+}$is accepted by the filter $\left.F\right\}, S=\left\{k \mid x_{k+1}=x_{k}+d_{k}\right\}$. Then, we have $S=\left\{k \mid \hat{\rho}_{k} \geq \mu_{1}\right.$ or $x_{k}^{+}$is accepted by the filter $\left.F\right\}$. When $k \notin S$, we have $x_{k+1}=x_{k}+\alpha_{k} d_{k}$.

\section{Convergence Analysis}

To establish the convergence of Algorithm 1, we make the following common assumption.

Assumption 1. H1. The level set $L\left(x_{0}\right)=\left\{x \in R^{n} \mid f(x) \leq f\left(x_{0}\right)\right\} \subset \Omega$, where $\Omega \in R^{n}$ is bounded $f(x)$ is continuously differentiable on the level set $L\left(x_{0}\right)$.

H2. The matrix $B_{k}$ is uniformly bounded, i.e., there exists a constant $M_{1}>0$ such that $\left\|B_{k}\right\| \leq M_{1}$.

H3. There exists constant $v$ such that $v\|d\|^{2} \leq d^{T} B_{k} d$, for all $k \in N \cup\{0\}$.

Remark 1. In order to analyze the convergence of the new algorithm, it should be seen to that the trial step $d_{k}$ satisfies the following conditions:

$$
\begin{gathered}
m_{k}(0)-m_{k}\left(d_{k}\right) \geq \tau\left\|g_{k}\right\| \min \left\{\Delta_{k}, \frac{\left\|g_{k}\right\|}{\left\|B_{k}\right\|}\right\}, \\
g_{k}^{T} d_{k} \leq-\tau\left\|g_{k}\right\| \min \left\{\Delta_{k}, \frac{\left\|g_{k}\right\|}{\left\|B_{k}\right\|}\right\},
\end{gathered}
$$

where the constant $\tau \in(0,1)$.

Remark 2. If $f$ is a twice continuously differentiable function, then $H 1$ means that there is a positive constant $L$ such that

$$
\|\nabla f(x)-\nabla f(y)\| \leq L\|x-y\| \forall x, y \in \Omega
$$

Lemma 1. For all $k$, we can obtain that

$$
\left|f_{k}-f\left(x_{k}+d_{k}\right)-\left(m_{k}(0)-m_{k}\left(d_{k}\right)\right)\right| \leq O\left(\left\|d_{k}\right\|^{2}\right),
$$


Proof. The proof is given by using Taylor's expansion and H3.

Lemma 2. Suppose that H1-H3 hold, the sequence $\left\{x_{k}\right\}$ is generated by Algorithm 1. Moreover, assume that there exists a constant $0<\varepsilon<1$ such that $\left\|g_{k}\right\|>\varepsilon$, for all $k$. Then, for every $k$, there exists a nonnegative integer $p$, so that $x_{k+p+1}$ is a successful iteration point, i.e., $\hat{\rho}_{k+p} \geq \mu_{1}$.

Proof. We are able to prove this by using contradiction; we assume that there exists an iteration $k$, and that $x_{k+p+1}$ is an unsuccessful iteration point for all nonnegative integer $p$, i.e.,

$$
\hat{\rho}_{k+p}<\mu_{1} p=0,1,2, \ldots
$$

It follows from (11) that $\hat{\rho}_{k+p^{\prime}}<\mu_{1} p=0,1,2, \ldots$ Thus, using (10) and (12), we show

$$
\Delta_{k+p+1} \leq \beta_{1}^{p+1} c_{k}\left\|g_{k+p}\right\|^{\gamma} \leq \beta_{1}^{p+1} c_{\max }\left\|g_{k}\right\|^{\gamma} .
$$

As a matter of fact, in the unsuccessful iterations, we have $x_{k+p}=x_{k}$. Thus, according to $0<\beta_{1}<1$ and (21), we have

$$
\lim _{p \rightarrow \infty} \Delta_{k+p+1}=0
$$

Now, using Lemma 1 and (16) we get

$$
\begin{aligned}
\left|\frac{f\left(x_{k+p}\right)-f\left(x_{k+p}+d_{k+p}\right)}{m_{k+p}(0)-m_{k+p}\left(d_{k+p}\right)}-1\right| & =\left|\frac{f\left(x_{k+p}\right)-f\left(x_{k+p}+d_{k+p}\right)-\left(m_{k+p}(0)-m_{k+p}\left(d_{k+p}\right)\right)}{m_{k+p}(0)-m_{k+p}\left(d_{k+p}\right)}\right| \\
& \left.\leq \frac{O\left(\left\|d_{k+p}\right\|^{2}\right)}{\tau\left\|g_{k+p}\right\| \min \left\{\Delta_{k+p},\left\|g_{k+p}\right\|\right.}\right\} \\
& \leq \frac{O\left(\left\|d_{k+p}\right\|^{2}\right)}{\tau \varepsilon \min \left\{\Delta_{k+p}, \frac{\varepsilon}{M_{1}}\right\}} \\
& \leq \frac{O\left(\Delta_{k+p}{ }^{2}\right)}{O\left(\Delta_{k+p}\right)} \rightarrow 0(p \rightarrow \infty)
\end{aligned}
$$

According to the definition of $R_{k}$, we get $R_{k} \geq \eta_{k} f_{k}+\left(1-\eta_{k}\right) f_{k}=f_{k}$. Thus, for sufficiently large $p$ we have,

$$
\hat{\rho}_{k+p}=\frac{R_{k+p}-f\left(x_{k+p}+d_{k+p}\right)}{m_{k+p}(0)-m_{k+p}\left(d_{k+p}\right)} \geq \frac{f_{k+p}-f\left(x_{k+p}+d_{k+p}\right)}{m_{k+p}(0)-m_{k+p}\left(d_{k+p}\right)} \rightarrow 1,
$$

which contradicts (20). This completes the proof of Lemma 2.

Lemma 3. Suppose that H1-H3 hold and the sequence $\left\{x_{k}\right\}$ is generated by Algorithm 1. Set $\delta \in\left(0, \min \left\{1, \frac{v}{L}\right\}\right)$. For $k \notin S$, we have

$$
f_{k+1}-R_{k} \leq \frac{\delta}{2}\left(1-\frac{L \delta}{v}\right) g_{k}^{T} d_{k} \leq 0
$$

Proof. According to the definition of $R_{k}$, for all $k$, we have $f_{k} \leq R_{k}$. Using the differential mean value theorem, we get

$$
f_{k+1}-R_{k} \leq f_{k+1}-f_{k}=g(\xi)^{T}\left(x_{k+1}-x_{k}\right),
$$

where $\xi \in\left[x_{k}, x_{k+1}\right]$. For $k \notin S$ and (18), we obtain 


$$
\begin{aligned}
g(\xi)^{T}\left(x_{k+1}-x_{k}\right) & =g_{k}^{T}\left(x_{k+1}-x_{k}\right)+\left(g(\xi)-g_{k}\right)^{T}\left(x_{k+1}-x_{k}\right) \\
& \leq g_{k}^{T}\left(x_{k+1}-x_{k}\right)+\left\|g(\xi)-g_{k}\right\|\left\|x_{k+1}-x_{k}\right\| \\
& \leq g_{k}^{T}\left(x_{k+1}-x_{k}\right)+L\left\|x_{k+1}-x_{k}\right\|^{2} \\
& =g_{k}^{T} \alpha_{k} d_{k}+L \alpha_{k}{ }^{2}\left\|d_{k}\right\|^{2} \\
& =\left(1-L \delta\left\|d_{k}\right\|^{2} / d_{k}^{T} B_{k} d_{k}\right) \alpha_{k} g_{k}^{T} d_{k} \\
& \leq(1-L \delta / v) \alpha_{k} g_{k}^{T} d_{k}
\end{aligned}
$$

Note that (4) and (16) imply that $\alpha_{k} \geq \frac{\delta}{2}$ for all. Setting $\delta \in\left(0, \min \left\{1, \frac{v}{L}\right\}\right)$ means that $1-L \delta / v>0$. According to (17), (25), and (26), we can conclude that (24) holds.

Lemma 4. Suppose that the sequence $\left\{x_{k}\right\}$ is generated by Algorithm 1. Then, we have $\left\{x_{k}\right\} \subset L\left(x_{0}\right)$.

Proof. We can proceed by induction. When $k=0$, apparently $x_{0} \in L\left(x_{0}\right)$.

Assuming that $x_{k} \in L\left(x_{0}\right)(k \geq 0)$ holds, we would obtain $f_{k} \leq f_{0}$. Then, we can prove that $x_{k+1} \in L\left(x_{0}\right)$.

(a) When $k \in S$, consider two cases:

Case 1: $k \in D$. According to (7) and (16), we obtain $R_{k}-f_{k+1} \geq \mu_{1}\left(m_{k}(0)-m_{k}\left(d_{k}\right)\right) \geq 0$. Thus, we have $R_{k} \geq f_{k+1}$. Following the definition of $R_{k}$ and $f_{l(k)}$, we obtain

$$
R_{k}=\eta_{k} f_{l(k)}+\left(1-\eta_{k}\right) f_{k} \leq \eta_{k} f_{l(k)}+\left(1-\eta_{k}\right) f_{l(k)}=f_{l(k)} .
$$

The above two inequalities show that

$$
f_{k+1} \leq R_{k} \leq f_{l(k)} \leq f_{0} .
$$

Case 2: $k \in A$. According to $0<\hat{\rho}_{k}<\mu_{1}$, we have $R_{k}-f\left(x_{k}+d_{k}\right)>0$. Thus, we get $f_{k+1} \leq R_{k} \leq f_{l(k)} \leq f_{0}$. (b) When $k \notin S$. Using Lemma 3 and (27), we obtain $f_{k+1} \leq R_{k} \leq f_{l(k)} \leq f_{0}$.

Now, we can conclude that $\left\{x_{k}\right\} \subset L\left(x_{0}\right)$.

Lemma 5. Suppose that H1-H3 hold, and the sequence $\left\{x_{k}\right\}$ is generated by Algorithm 1. This would mean that $\left\{f_{l(k)}\right\}$ is a not a monotonically increasing sequence, nor is it convergent.

Proof. Now, we can prove that the sequence $\left\{f_{l(k)}\right\}$ is not a monotonically increasing sequence. Thus, we consider two cases:

Case 1: For $k<N$, it is clear that $m(k)=k$. Since, for any $k$, we have $f_{k} \leq f_{0}$. Thus, we get $f_{l(k)}=f_{0}$. Case 2: For $k \geq N$, we obtain $m(k+1)=m(k)+1$. Thus, using the definition of $f_{l(k+1)}$ and (5), we observe that

$$
f_{l(k+1)}=\max _{0 \leq j \leq m(k+1)}\left\{f_{k+1-j}\right\} \leq \max _{0 \leq j \leq m(k)+1}\left\{f_{k+1-j}\right\}=\max \left\{f_{l(k)}, f_{k+1}\right\} \leq f_{l(k)},
$$

$\left\{f_{l(k)}\right\}$ is not a monotonically increasing sequence. This fact, along with $\mathrm{H} 1$, implies that

$$
\exists \lambda \text {, s.t. } \forall n \in N \cup\{0\}: \lambda \leq f_{k+n} \leq f_{l(k+n)} \leq \ldots \leq f_{l(k+1)} \leq f_{l(k)} .
$$

This shows that the sequence $\left\{f_{l(k)}\right\}$ is convergent.

Lemma 6. Suppose that H1-H3 hold, and there exists $\varepsilon>0$ such that $\left\|g_{k}\right\| \geq \varepsilon$, for all $k$. Then, there is a constant $\beta>0$, and we have

$$
\Delta_{k} \geq \frac{\beta}{M_{k}}
$$


where $M_{k}=\max _{0 \leq i \leq k}\left\|B_{i}\right\|+1$.

Proof. Set $\sigma=\frac{\tau \varepsilon\left(1-\mu_{1}\right)}{2 L}$. We proceed by induction; set

$$
\beta=\left\{\Delta_{0} M_{0}, \beta_{1} \sigma M_{0},\left(1-\mu_{1}\right) \beta_{1} \tau \varepsilon, \beta_{1} \varepsilon\right\},
$$

When $k=0$, we can see that $\Delta_{0} \geq \frac{\beta}{M_{0}}$. Then, assume that (31) holds for $k$. Note that $\left\{M_{k}\right\}$ is an increasing sequence. Thus, we prove that

$$
\Delta_{k+1} \geq \frac{\beta}{M_{k}}, \quad k=0,1, \ldots,
$$

(a) When $k \in D$, i.e., $\hat{\rho}_{k} \geq \mu_{1}$. Using (11) and (7), we deduce that $\hat{\rho}_{k^{\prime}} \geq \mu_{1}$. From (12), we get $\Delta_{k+1}=\lambda \Delta_{k}$, where $\lambda>1$ is a constant. Thus, the inequality $\Delta_{k} \geq \frac{\beta}{M_{k}}$ implies that $\Delta_{k+1} \geq \frac{\beta}{M_{k}}$.

(b) When $k \in A$, i.e., $0<\hat{\rho}_{k}<\mu_{1}$. Supposing that $\left\|d_{k}\right\| \geq \sigma$, we have

$$
\Delta_{k+1}=\beta_{1} \Delta_{k} \geq \beta_{1}\left\|d_{k}\right\| \geq \beta_{1} \sigma \geq \frac{\beta_{1} \sigma M_{0}}{M_{k}} \geq \frac{\beta}{M_{k}}
$$

Then, assuming that $\left\|d_{k}\right\|<\sigma$, we have

$$
\frac{f_{k}-f\left(x_{k}+d_{k}\right)}{m_{k}(0)-m_{k}\left(d_{k}\right)} \leq \frac{R_{k}-f\left(x_{k}+d_{k}\right)}{m_{k}(0)-m_{k}\left(d_{k}\right)}<\mu_{1}
$$

Thus,

$$
f\left(x_{k}+d_{k}\right)-f_{k}>-\mu_{1}\left(m_{k}(0)-m_{k}\left(d_{k}\right)\right)=\mu_{1}\left(g_{k}^{T} d_{k}+\frac{1}{2} d_{k}^{T} B_{k} d_{k}\right) .
$$

Using Taylor's formula and H1-H3, it is easy to show that

$$
\begin{aligned}
f\left(x_{k}+d_{k}\right)-f_{k} & \leq g(\eta)^{T} d_{k}=g_{k}^{T} d_{k}+\left(g(\eta)-g_{k}\right)^{T} d_{k} \\
& \leq g_{k}^{T} d_{k}+L\left\|d_{k}\right\|^{2} \\
& \leq g_{k}^{T} d_{k}+\frac{\tau\left(1-\mu_{1}\right) \varepsilon\left\|d_{k}\right\|}{2}
\end{aligned}
$$

where $\eta \in\left[x_{k}, x_{k}+d_{k}\right]$. When combining (35) with (36), we discover that

$$
(1-\mu)\left(g_{k}^{T} d_{k}+\frac{\tau \varepsilon\left\|d_{k}\right\|}{2}\right)>\frac{\mu d_{k}^{T} B_{k} d_{k}}{2} .
$$

Moreover, the inequality (16), together with $\left\|g_{k}\right\| \geq \varepsilon$, imply that

$$
-g_{k}^{T} d_{k}-\frac{1}{2} d_{k}^{T} B_{k} d_{k} \geq \tau \varepsilon \min \left\{\Delta_{k}, \frac{\varepsilon}{\left\|B_{k}\right\|}\right\} .
$$

Multiply the two sides of inequality (38) by $(1-\mu)$, such that

$$
-(1-\mu)\left(g_{k}^{T} d_{k}+d_{k}^{T} B_{k} d_{k} / 2\right) \geq\left(1-\mu_{1}\right) \tau \varepsilon \min \left\{\left\|\Delta_{k}\right\|, \frac{\varepsilon}{\left\|B_{k}\right\|}\right\} .
$$

On the other hand, from H3, (37), and (39), we have

$$
\Delta_{k}\left\|B_{k}\right\| \geq \tau\left(1-\mu_{1}\right) \varepsilon \min \left\{1, \frac{2 \varepsilon}{\left\|B_{k}\right\| \Delta_{k}}-1\right\}
$$


If $\Delta_{k}\left\|B_{k}\right\| \leq \varepsilon$, we have $\Delta_{k}\left\|B_{k}\right\|>(1-\mu) \tau \varepsilon$. Otherwise, we obtain $\Delta_{k}\left\|B_{k}\right\|>\varepsilon$. Now, following (40) we obtain

$$
\Delta_{k}\left\|B_{k}\right\| \geq \min \left\{\left(1-\mu_{1}\right) \tau \varepsilon, \varepsilon\right\}
$$

Thus,

$$
\Delta_{k+1}=\beta_{1} \Delta_{k} \geq \frac{\min \left\{\left(1-\mu_{1}\right) \beta_{1} \tau \varepsilon, \beta_{1} \varepsilon\right\}}{\left\|B_{k}\right\|} \geq \frac{\beta}{M_{k}}
$$

The proof is completed.

Based on the analyses and lemmas above, we obtain the global convergence of Algorithm 1 as follows:

Theorem 1. (Global Convergence) Suppose that H1-H3 hold, and the sequence $\left\{x_{k}\right\}$ is generated by Algorithm 1. Then,

$$
\liminf _{k \rightarrow \infty}\left\|g_{k}\right\|=0
$$

Proof. Consider the following two cases:

Case 1: The number of successful iterations and many filter iterations are infinite, e.g., $|S|=+\infty$, $|A|=+\infty$.

We proceed from this proof with a contradiction. Suppose that (42) is not true, then there exists a positive constant $\varepsilon$ such that $\left\|g_{k}\right\|>\varepsilon$. From H1, we can see that $\left\{\left\|g_{k}\right\|\right\}$ is bounded. Set in the index of set $A$ is the sequence $\left\{k_{i}\right\}$. Thus, there exists a subsequence $\left\{k_{t}\right\} \subseteq\left\{k_{i}\right\}$, which satisfies $\lim _{t \rightarrow \infty} g_{k_{t}}=g_{\infty}$, $g_{\infty} \geq \varepsilon, \exists j \in\{1,2, \ldots, n\}$ and we have

$$
\left|g_{k_{t}}^{j}\right|-\left|g_{k_{t-1}}^{j}\right| \leq-\gamma_{g}\left\|g_{k_{t-1}}\right\|
$$

Using (43), as $t$ is sufficiently large, we have

$$
0 \leftarrow\left|g_{k_{t}}^{j}\right|-\left|g_{k_{t-1}}^{j}\right| \leq-\gamma_{g} \varepsilon<0
$$

As this is a contradiction, the proof is completed.

Case 2: The number of successful iterations is infinite, and the number of filter iterations is finite, e.g., $|S|=+\infty,|A|<+\infty$.

Assume for a moment that there exists an integer constant $k_{1}$, such that $k>k_{1}$. This implies that $k \in D$, and we therefore have $\hat{\rho}_{k} \geq \mu_{1}$. Hence, from (16) and (27), we find that

$$
f_{l(k)}-f_{k+1} \geq R_{k}-f_{k+1} \geq \mu_{1} \tau\left\|g_{k}\right\| \min \left\{\Delta_{k}, \frac{\left\|g_{k}\right\|}{\left\|B_{k}\right\|}\right\} \geq 0
$$

We proceed from this proof with a contradiction. Suppose that there exist constants $\varepsilon>0$ and $k_{2}>k_{1}$, such that $\left\|g_{k}\right\| \geq \varepsilon, \forall k \geq k_{2}$. Based on Lemma 6 and (45), we write

$$
f_{l(k)}-f_{k+1} \geq R_{k}-f_{k+1} \geq \mu_{1} \tau \varepsilon \min \left\{\frac{\beta}{M_{k}}, \frac{\varepsilon}{M_{k}}\right\}=\mu_{1} \tau \varepsilon \frac{\min \{\beta, \varepsilon\}}{M_{k}} .
$$

Set $a=\mu_{1} \tau \varepsilon \min \{\beta, \varepsilon\}$, thus,

$$
f_{l(k)}-f_{k+1} \geq \frac{a}{M_{k}}
$$


According to (47) and $\left\{M_{k}\right\}$, this is an increasing sequence, as we have

$$
\begin{aligned}
& f_{l(k)} \geq f_{k+1}+a / M_{k} \geq f_{k+1}+a / M_{k+M+1} \\
& f_{l(k+1)} \geq f_{k+2}+a / M_{k+1} \geq f_{k+2}+a / M_{k+M+1} \\
& \begin{array}{llll}
\vdots & \vdots & \vdots & \vdots
\end{array} \\
& f_{l(k+M)} \geq f_{k+M+1}+a / M_{k+M} \geq f_{k+M+1}+a / M_{k+M+1}
\end{aligned}
$$

We then take the maximum value of both sides of (48), along with Lemma 5, to imply that

$$
f_{l(k)} \geq \max \left\{f_{k+1}, f_{k+2}, \ldots, f_{k+M+1}\right\}+a / M_{k+M+1} \quad \forall k \geq k_{2} .
$$

According to (5), we have

$$
f_{l(k+M+1)} \leq \max \left\{f_{k+1}, f_{k+2}, \ldots, f_{k+M+1}\right\}
$$

Thus, we get

$$
f_{l(k)}-f_{l(k+M+1)} \geq a / M_{k+M+1} .
$$

Now, using (51), we deduce that

$$
\begin{aligned}
\sum_{k \geq k_{2}} \frac{1}{M_{k+M+1}} & \leq \frac{1}{a} \sum_{k \geq k_{2}}\left(f_{l(k)}-f_{l(k+M+1)}\right) \\
& =\frac{1}{a} \sum_{k \geq k_{2}} \sum_{s=0}^{M}\left(f_{l(k+s)}-f_{l(k+s+1)}\right) \\
& =\frac{1}{a} \sum_{k \geq k_{2}}\left(f_{l(k)}-f_{l(k+1)}\right)<+\infty
\end{aligned}
$$

which contradicts $\sum_{k=1}^{\infty} \frac{1}{M_{k}}=+\infty$. This completes the proof of Theorem 1 .

\section{Local Convergence}

In this section, we will demonstrate the superlinear convergence of Algorithm 1 under appropriate conditions.

Theorem 2. (Superlinear Convergence) Suppose that H1-H3 hold, and the sequence $\left\{x_{k}\right\}$ generated by Algorithm 1 converges to $x^{*}$. Moreover, assume that $\nabla^{2} f\left(x^{*}\right)$ is a positive definite, and $\nabla^{2} f(x)$ is Lipschitz continuous in a neighborhood of $x^{*}$. If $\left\|d_{k}\right\| \leq \Delta_{k}$, where $d_{k}=-B_{k}^{-1} g_{k}$, and

$$
\lim _{k \rightarrow \infty} \frac{\|\left(B_{k}-\nabla^{2} f\left(x^{*}\right) d_{k} \|\right.}{\left\|d_{k}\right\|}=0
$$

Then, the sequence $\left\{x_{k}\right\}$ converges to $x^{*}$ superlinearly, that is,

$$
\left\|x_{k+1}-x^{*}\right\|=o\left(\left\|x_{k}-x^{*}\right\|\right)
$$

Proof. Following Lemmas 1 and 2 , it is obvious that $\hat{\rho}_{k} \geq \mu_{1}$ for sufficiently large $k$. This shows that Algorithm 1 has been simplified to the superlinear convergence standard quasi-Newton methods [22]. Thus, the superlinear convergence of this algorithm can be proven to be similar to Theorem 5.5.1 in [22]. We omit it for convenience. 


\section{Preliminary Numerical Experiments}

In this section, we perform numerical experiments on Algorithm 1, and compare it with Mo [1] and Hang [4]. A set of unconstrained test problems (of variable dimension) are selected from [23]. The simulation experiment uses MATLAB 9.4 and the processor uses Intel (R) Core (TM), $2.00 \mathrm{GHz}, 6 \mathrm{~GB}$ RAM. Take exactly the same value for the public parameters of these algorithms: $\mu_{1}=0.25, \mu_{2}=0.75$, $\beta_{1}=0.25, \beta_{2}=1.5, M=5$. In our experiments, algorithms are being stopped when $\left\|g_{k}\right\| \leq 10^{-6}\left\|g_{0}\right\|$ or the number of iterations exceeds 10,000. We denote the running time via CPU. $n_{f}$ and $n_{i}$ denoted the total number of gradient evaluations, and the total number of function evaluations, respectively. The matrix $B_{k}$ is updated using a MBFGS formula [24]:

$$
B_{k+1}=\left\{\begin{array}{cc}
B_{k}+\frac{z_{k} z_{k}^{T}}{z_{k}^{T} d_{k}}-\frac{B_{k} d_{k} d_{k}^{T} B_{k}}{d_{k}^{T} B_{k} d_{k}}, & y_{k}^{T} d_{k}>0 \\
B_{k}, & y_{k}^{T} d_{k} \leq 0
\end{array}\right.
$$

where $d_{k}=x_{k+1}-x_{k}, y_{k}=g_{k+1}-g_{k}, z_{k}=y_{k}+t_{k}\left\|g_{k}\right\| d_{k}, t_{k}=1+\max \left\{-\frac{y_{k}^{T} d_{k}}{\left\|g_{k}\right\|\left\|d_{k}\right\|}, 0\right\}$.

To facilitate this, we used the following notations to represent the algorithms:

ANTRFS: A Nonmonotone Trust Region Method with Fixed Step length [1];

FSNATR: On a Fixed Step length Nonmonotone Adaptive Trust Region Method [4];

From Table 1, there are some variable dimension problems, which select the dimension in the range $[4,1000]$. We know that the new algorithm is generally better than NTRFS and FSNATR in terms of the total number of gradient evaluations and function evaluations. The new algorithm solves all the test functions in Table 1. The performance profiles given by Dolan and Moré [25] are used to compare the efficiency of the three algorithms. Figures 1-3 give the performance profiles of the three algorithms for running time, the number of gradient evaluations, and the number of function evaluations, respectively. The figures show that Algorithm 1 performs well when compared with the other algorithms, at least on the test problems considered, which are mostly of small dimension. It can be observed that Algorithm 1 increases faster than the other algorithms, especially in contrast to NTRFS. Therefore, we can deduce that the new algorithm is more efficient and robust than the other considered trust region algorithms for solving small and medium-scale unconstrained optimization problems.

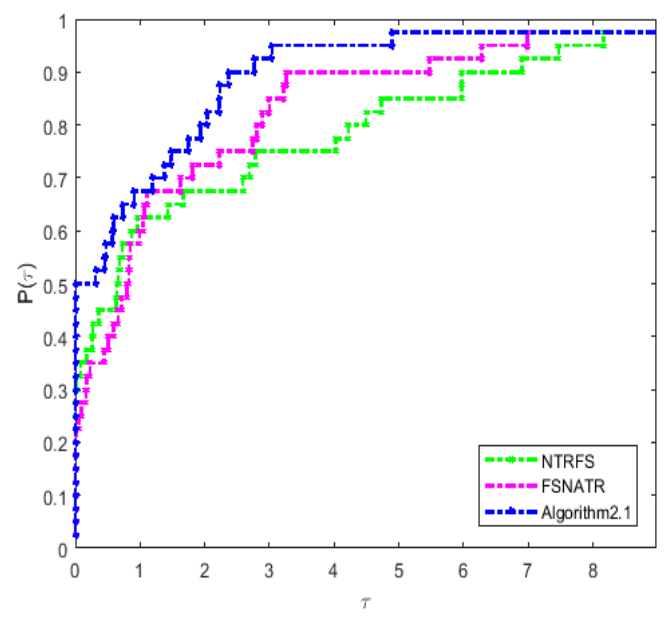

Figure 1. CPU performance profile for the three algorithms. 
Table 1. Numerical comparisons on a subset of test problems.

\begin{tabular}{|c|c|c|c|c|c|c|c|}
\hline Problem & $n$ & $n_{f} / n_{i}$ & & & & & \\
\hline & & ANTRFS [9] & CPU & FSNATR [20] & CPU & Algorithm 1 & CPU \\
\hline Ext.Rose & 4 & $755 / 382$ & 2.755795 & $168 / 88$ & 0.322036 & $87 / 58$ & 0.104316 \\
\hline Ext. Beale & 4 & $25 / 13$ & 0.008651 & $41 / 21$ & 0.069185 & $18 / 16$ & 0.028946 \\
\hline Penalty i & 2 & $18 / 10$ & 0.087532 & $18 / 10$ & 0.067020 & $17 / 14$ & 0.032533 \\
\hline Pert.Quad & 6 & $28 / 25$ & 0.058921 & $25 / 13$ & 0.058700 & $18 / 17$ & 0.035631 \\
\hline Raydan 1 & 8 & $18 / 10$ & 0.015109 & $38 / 20$ & 0.105928 & $39 / 20$ & 0.070292 \\
\hline Raydan 2 & 4 & $21 / 11$ & 0.015356 & $13 / 8$ & 0.012729 & $11 / 6$ & 0.017449 \\
\hline Diagonal 1 & 10 & $13 / 8$ & 0.009493 & $35 / 18$ & 0.070199 & $27 / 26$ & 0.064282 \\
\hline Diagonal 2 & 10 & $56 / 29$ & 0.017841 & $58 / 30$ & 0.119385 & $57 / 29$ & 0.083905 \\
\hline Diagonal 3 & 50 & $200 / 101$ & 1.926143 & $182 / 92$ & 1.232287 & $127 / 126$ & 1.849887 \\
\hline Hager & 10 & $27 / 14$ & 0.049037 & $27 / 14$ & 0.048247 & $33 / 17$ & 0.071906 \\
\hline Gen. Trid 1 & 20 & $967 / 484$ & 3.536055 & $50 / 26$ & 0.432577 & $47 / 24$ & 0.217367 \\
\hline Ext.Trid 1 & 10 & $27 / 14$ & 0.013890 & $29 / 15$ & 0.128696 & $18 / 12$ & 0.071580 \\
\hline Ext. TET & 50 & $13 / 7$ & 0.203093 & $16 / 9$ & 0.031416 & $17 / 9$ & 0.119907 \\
\hline Diadonal 4 & 100 & $7 / 4$ & 0.035933 & $9 / 5$ & 0.343849 & $5 / 4$ & 0.146901 \\
\hline Ext.Him & 100 & $29 / 15$ & 0.147102 & $25 / 13$ & 0.208976 & $29 / 28$ & 0.409463 \\
\hline Gen. White & 50 & $785 / 576$ & 10.47342 & $771 / 429$ & 9.940880 & $443 / 228$ & 5.741535 \\
\hline Ext. Powell & 16 & $1567 / 787$ & 7.266044 & $794 / 404$ & 2.148929 & $496 / 337$ & 1.208253 \\
\hline Full Hessian FH3 & 100 & $11 / 6$ & 0.053598 & $11 / 6$ & 0.084726 & $8 / 7$ & 0.088831 \\
\hline Ext.BD1 & 100 & $51 / 27$ & 0.210790 & $50 / 28$ & 0.739621 & $21 / 15$ & 0.261978 \\
\hline Pert. Quad & 200 & $91 / 66$ & 2.547689 & $87 / 44$ & 2.421596 & $57 / 56$ & 2.405979 \\
\hline Extended Hiebert & 16 & $1821 / 1000$ & 9.819290 & $175 / 143$ & 2.456780 & $135 / 68$ & 0.527388 \\
\hline Quadratic QF1 & 4 & $15 / 8$ & 0.007903 & $17 / 9$ & 0.017025 & $11 / 10$ & 0.010983 \\
\hline FLETCHCR34 & 36 & $210 / 123$ & 1.847519 & $150 / 91$ & 0.950314 & $165 / 83$ & 1.786160 \\
\hline ARWHEAD & 200 & $297 / 150$ & 37.928050 & $29 / 15$ & 0.317976 & $15 / 12$ & 0.317976 \\
\hline NONDIA & 50 & $75 / 39$ & 0.368280 & $92 / 47$ & 0.544079 & $51 / 35$ & 0.307129 \\
\hline DQDRTIC & 50 & $67 / 38$ & 0.51243 & $53 / 28$ & 0.341435 & $32 / 30$ & 0.318596 \\
\hline EG2 & 200 & $32 / 17$ & 0.319954 & $28 / 16$ & 0.373764 & $49 / 35$ & 2.633184 \\
\hline Bro.Tridiagonal & 200 & $2797 / 1504$ & 441.453385 & $744 / 398$ & 119.570838 & $69 / 35$ & 1.539657 \\
\hline A.Per.Quad & 16 & $73 / 47$ & 0.144890 & $63 / 32$ & 0.132644 & $45 / 26$ & 0.128349 \\
\hline Pert.Trid.Quad & 100 & $330 / 166$ & 10.985321 & $325 / 163$ & 9.663929 & $289 / 156$ & 8.521700 \\
\hline Ext.DENSCH & 100 & $37 / 19$ & 0.190549 & $43 / 22$ & 0.398777 & $128 / 82$ & 5.638770 \\
\hline SINCOS & 100 & $4303 / 2152$ & 198.717544 & $1303 / 952$ & 142.543185 & $65 / 36$ & 1.122092 \\
\hline BIGGSB1 & 10 & $1949 / 1042$ & 8.466655 & $329 / 195$ & 0.676394 & $275 / 185$ & 0.376394 \\
\hline ENGVAL1 & 200 & $788 / 487$ & 139.949938 & $643 / 406$ & 99.088596 & $474 / 472$ & 88.401960 \\
\hline EDENSCH & 100 & $474 / 238$ & 25.639664 & $45 / 26$ & 0.407574 & $37 / 23$ & 0.930150 \\
\hline CUBE & 100 & $430 / 220$ & 21.53234 & $357 / 198$ & 20.93564 & $280 / 147$ & 13.946540 \\
\hline BDEXP & 100 & $476 / 369$ & 34.54797 & $452 / 356$ & 24.569196 & $22 / 21$ & 0.550708 \\
\hline GENHUMPS & 100 & $532 / 321$ & 3.27453 & $412 / 213$ & 0.475453 & $1014 / 537$ & 1.235720 \\
\hline QUARTC & 100 & $57 / 32$ & 1.035734 & $43 / 22$ & 0.443325 & $18 / 17$ & 0.326680 \\
\hline Gen. PSC1 & 500 & $198 / 212$ & 10.457624 & $51 / 54$ & 9.562354 & $51 / 54$ & 8.539801 \\
\hline Ext. PSC1 & 500 & $15 / 15$ & 1.254327 & $15 / 15$ & 1.0983452 & $13 / 13$ & 1.562763 \\
\hline Variably dim. & 500 & $41 / 27$ & 2.9578243 & $21 / 16$ & 1.4536982 & $17 / 15$ & 1.093456 \\
\hline DIXMAANA & 1000 & $21 / 21$ & 1.457893 & $21 / 21$ & 1.237642 & $20 / 20$ & 1.025372 \\
\hline SINQUAD & 1000 & $1582 / 1063$ & 187.563723 & $1995 / 1215$ & 135.872354 & $912 / 579$ & 100.458723 \\
\hline DIXMAANJ & 1000 & $2415 / 2398$ & 431.253485 & $2320 / 2311$ & 410.253485 & $2246 / 2132$ & 397.256732 \\
\hline
\end{tabular}

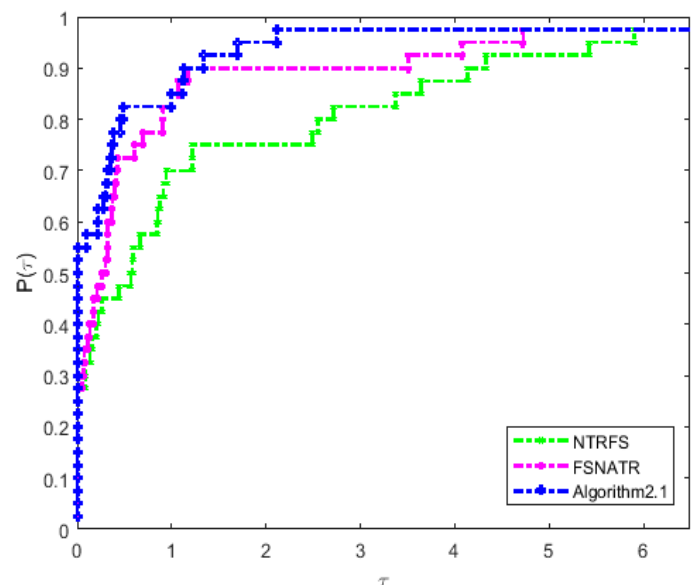

Figure 2. Performance profile for the number of gradient evaluations $\left(n_{i}\right)$. 


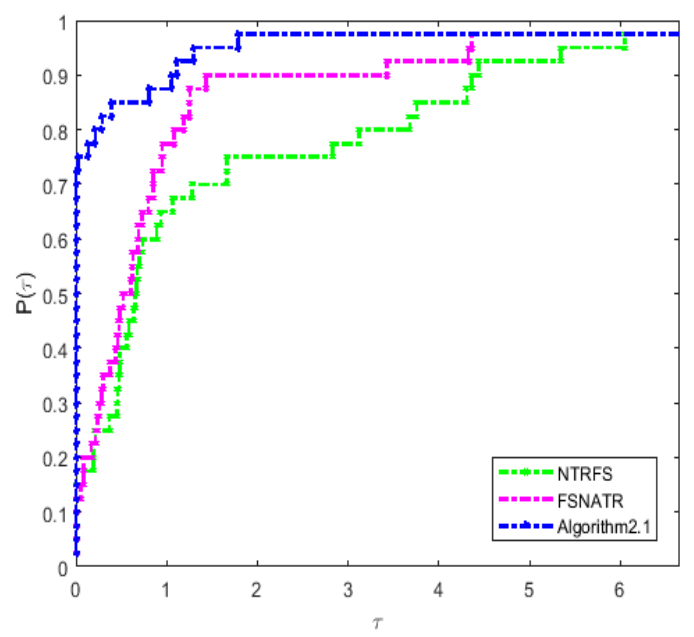

Figure 3. Performance profile for the number of function evaluations $\left(n_{f}\right)$.

\section{Conclusions}

In this paper, the authors proposed a new nonmonotone trust region method and also put forward the following innovations:

(1) a new adaptive radius strategy to reduce the number of calculations;

(2) a modified trust region ratio to solve effectively unconstrained optimization problems. The filter technology is also important. Theorems 1 and 2 show that the proposed algorithm can preserve global convergence and superlinear convergence, respectively. According to preliminary numerical experiments, we can conclude that the new algorithm is very effective for unconstrained optimization, and the nonmonotone technology is very helpful for many optimization problems. In the future, we will have more ideas for solving many optimization problems, such as combining the modified conjugate gradient algorithm with a modified trust region method. We can also use the new algorithm for solving constrained optimization problems.

Author Contributions: Conceptualization, X.W. and Q.Q.; methodology, X.W.; software, X.W.; validation, X.W., Q.Q. and X.D.; formal analysis, X.D.; investigation, Q.Q.; resources, X.D.; data curation, Q.Q.; writing-original draft preparation, X.W.; writing-review and editing, X.W. All authors have read and agreed to the published version of the manuscript.

Funding: This research received no external funding.

Acknowledgments: At the point of finishing this paper, I'd like to express my sincere thanks to all those who have lent me a hand over the course of my writing this paper. First of all, I would like to take this opportunity to show my sincere gratitude to my supervisor, Xianfeng Ding, who has given me so much useful advice on my writing and has tried his best to improve my paper. Secondly, I would like to express my gratitude to my classmates, who offered me references and information on time. Without their help, it would have been much harder for me to finish this paper.

Conflicts of Interest: The authors declare no conflict of interest.

\section{References}

1. Mo, J.T.; Zhang, K.C.; Wei, Z.X. A nonmonotone trust region method for unconstrained optimization. Appl. Math. Comput. 2005, 171, 371-384. [CrossRef]

2. Ou, Y.G.; Zhou, Q.; Lin, H.C. An ODE-based trust region method for unconstrained optimization problems. J. Comput. Appl. Math. 2009, 232, 318-326. [CrossRef]

3. Wang, X.Y.; Tong, J. A Nonmonotone Adaptive Trust Region Algorithm with Fixed Stepsize for Unconstrained Optimization Problems. Math. Appl. 2009, 3, 496-500.

4. Hang, D.; Liu, M. On a Fixed Stepsize Nonmonotonic Self-Adaptive Trust Region Algorithm. J. Southwest China Norm. Univ. 2013, 38. [CrossRef] 
5. Sartenaer, A. Automatic determination of an initial trust region in nonlinear programming. SIAM J. Sci. Comput. 1997, 18, 1788-1803. [CrossRef]

6. Zhang, X.S.; Zhang, J.L.; Liao, L.Z. An adaptive trust region method and its convergence. Sci. China 2002, 45, 620-631. [CrossRef]

7. Shi, Z.J.; Guo, J.H. A new trust region methods for unconstrained optimization. Comput. Appl. Math. 2008, 213, 509-520. [CrossRef]

8. Kimiaei, M. A new class of nonmonotone adaptive trust-region methods for nonlinear equations with box constraints. Calcolo 2017, 54, 769-812. [CrossRef]

9. Amini, K.; Shiker Mushtak, A.K.; Kimiaei, M. A line search trust-region algorithm with nonmonotone adaptive radius for a system of nonlinear equations. Q. J. Oper. Res. 2016, 4, 132-152. [CrossRef]

10. Peyghami, M.R.; Tarzanagh, D.A. A relaxed nonmonotone adaptive trust region method for solving unconstrained optimization problems. Comput. Optim. Appl. 2015, 61, 321-341. [CrossRef]

11. Deng, N.Y.; Xiao, Y.; Zhou, F.J. Nonmonotone Trust Region Algorithm. J. Optim. Theory Appl. 1993, 76, 259-285. [CrossRef]

12. Ahookhoosh, M.; Amini, K.; Peyghami, M. A nonmonotone trust region line search method for large scale unconstrained optimization. Appl. Math. Model. 2012, 36, 478-487. [CrossRef]

13. Zhang, H.C.; Hager, W.W. A nonmonotone line search technique and its application to unconstrained optimization. SIAM J. Optim. 2004, 14, 1043-1056. [CrossRef]

14. Gu, N.Z.; Mo, J.T. Incorporating nonmonotone strategies into the trust region for unconstrained optimization. Comput. Math. Appl. 2008, 55, 2158-2172. [CrossRef]

15. Fletcher, R.; Leyffer, S. Nonlinear programming without a penalty function. Math. Program. 2002, 91, 239-269. [CrossRef]

16. Gould, N.I.; Sainvitu, C.; Toint, P.L. A filter-trust-region method for unconstrained optimization. SIAM J. Optim. 2005, 16, 341-357. [CrossRef]

17. Gould, N.I.; Leyffer, S.; Toint, P.L. A multidimensional filter algorithm for nonlinear equations and nonlinear least-squares. SIAM J. Optim. 2004, 15, 17-38. [CrossRef]

18. Wächter, A.; Biegler, L.T. Line search filter methods for nonlinear programming and global convergence. SIAM J. Optim. 2005, 16, 1-31. [CrossRef]

19. Miao, W.H.; Sun, W. A filter trust-region method for unconstrained optimization. Numer. Math. J. Chin. Univ. 2007, 19, 88-96.

20. Zhang, Y.; Sun, W.; Qi, L. A nonmonotone filter Barzilai-Borwein method for optimization. Asia Pac. J. Oper. Res. 2010, 27, 55-69. [CrossRef]

21. Fatemi, M.; Mahdavi-Amiri, N. A filter trust-region algorithm for unconstrained optimization with strong global convergence properties. Comput. Optim. Appl. 2012, 52, 239-266. [CrossRef]

22. Nocedal, J.; Wright, S.J. Numerical Optimization; Springer: New York, NY, USA, 2006.

23. Andrei, N. An unconstrained optimization test functions collection. Environ. Sci. Technol. 2008, 10, 6552-6558.

24. Pang, S.; Chen, L. A new family of nonmonotone trust region algorithm. Math. Pract. Theory. 2011, 10, 211-218.

25. Dolan, E.D.; Moŕe, J.J. Benchmarking optimization software with performance profiles. Math. Program. 2002, 91, 201-213. [CrossRef]

(C) 2020 by the authors. Licensee MDPI, Basel, Switzerland. This article is an open access article distributed under the terms and conditions of the Creative Commons Attribution (CC BY) license (http://creativecommons.org/licenses/by/4.0/). 